\title{
Mechanisms for the Cognitive Processing of Attractiveness in Adult and Infant Faces: From the Evolutionary Perspective
}

\author{
Hui Kou ${ }^{1}$, Qinhong $\mathrm{Xie}^{2}$ and Taiyong $\mathrm{Bi}^{1 *}$ \\ ${ }^{1}$ Center for Mental Health Research in School of Management, Zunyi Medical University, Guizhou, China, ${ }^{2}$ School \\ of Criminal Justice, China University of Political Science and Law, Beijing, China
}

OPEN ACCESS

Edited by:

Lin Zhang,

Ningbo University, China

Reviewed by:

Lijun Yin,

Sun Yat-sen University, China

Yan Zhang,

Huazhong University of Science and Technology, China

${ }^{*}$ Correspondence:

Taiyong $\mathrm{Bi}$

bitaiyong@126.com

Specialty section:

This article was submitted to Evolutionary Psychology, a section of the journal

Frontiers in Psychology

Received: 14 January 2020

Accepted: 25 February 2020

Published: 11 March 2020

Citation:

Kou H, Xie Q and Bi T (2020)

Mechanisms for the Cognitive Processing of Attractiveness in Adult and Infant Faces: From

the Evolutionary Perspective.

Front. Psychol. 11:436.

doi: 10.3389/fpsyg.2020.00436
Research on the cognitive processing of facial attractiveness has mainly focused on adult faces. Recent studies have revealed that the cognitive processing of facial attractiveness in infant faces is not the same as that in adult faces. Therefore, it is necessary to summarize the evidence on the processing of facial attractiveness in each kind of face and compare their underlying mechanisms. In this paper, we first reviewed studies on the cognitive processing of facial attractiveness in adult faces, including attentional and mnemonic processing, and then discussed the underlying mechanisms. Afterward, studies on facial attractiveness in infant faces were reviewed, and the underlying mechanisms were also discussed. Direct comparisons between the two kinds of cognitive processing were subsequently made. The results showed that the mechanisms for the processing of attractiveness in adult faces and infant faces are mainly motivated by the perspectives of mate selection and raising offspring, respectively, in evolutionary psychology. Finally, directions for future research are proposed.

Keywords: adult face, infant face, facial attractiveness, cognitive processing, evolutionary psychology

\section{INTRODUCTION}

Faces can be automatically evaluated on multiple trait dimensions. Through a dimensional reduction technique, varieties of traits of adult (Oosterhof and Todorov, 2008; Sutherland et al., 2013) and infant faces (Collova et al., 2019) were reduced to "valence/approachability" for adult faces and "niceness" for infant faces respectively. Facial attractiveness, which refers to the pleasant emotional experience and approaching intention induced by a human face (Rhodes, 2006), is closely associated to the general positivity/negativity in evaluations of adult and infant faces. Facial attractiveness is also proposed to be divided into two components: sexual attractiveness and cuteness (Rhodes, 2006). Later, Geldart (2010) proposed that female beauty can be mainly described by attractiveness, prettiness, beauty and cuteness. Evidently, attractiveness in adult faces may be mainly characterized by prettiness and beauty, while that in infant faces may be mainly characterized by cuteness. Evidence has shown that the evaluation of cuteness in a face is independent of that of attractiveness, prettiness and beauty (Geldart, 2010). Thus, there may be different mechanisms for the cognitive processing of attractiveness in adult faces and infant faces. However, studies on facial attractiveness mainly focus on adult faces. In the present review, 
we summarized and compared the cognitive processing of facial attractiveness in both adult and infant faces. Different and common underlying mechanisms were then discussed to provide a more comprehensive view of facial attractiveness.

\section{COGNITIVE PROCESSING OF FACIAL ATTRACTIVENESS IN ADULT FACES}

Studies on the cognitive processing of facial attractiveness in adult faces have mainly focused on attentional processing and mnemonic processing. The results are usually interpreted based on the perspective of mate selection in evolutionary psychology.

Highly attractive (HA) faces can attract visual attention (Sui and Liu, 2009; Lindell and Lindell, 2014), which is evident in newborns and infants (Slater et al., 2000; Van Duuren et al., 2003; Griffey and Little, 2014). Moreover, facial attractiveness may influence visual attention in several ways. Please note that the level of attentional bias induced by facial attractiveness may be different between men and women. We may discuss the influence of observers' sex in later paragraphs. In this paragraph, we only focus on the attentional bias induced by facial attractiveness regardless of observers' sex. First, compared with lowly attractive (LA) faces, individuals showed attentional maintenance toward and attentional disengagement from HA faces (Maner et al., 2007a,b; Silva et al., 2016). For example, studies of eye-movement tracking revealed that individuals showed a longer fixation duration on HA faces than that on LA faces (Shimojo et al., 2003; Dewall and Maner, 2008; Kou et al., 2016), which were also evident when faces were displayed in natural scenes (Leder et al., 2010, 2016; Mitrovic et al., 2016). Second, target-tracking behavior or the sustained attention can be modulated by the attractiveness of the target and distractor. For example, a recent study showed higher performance for the tracking of HA faces than for that of LA faces when participants were required to track moving faces among distractors and report the final location of each target ( $\mathrm{Li}$ et al., 2016). When HA faces were distractors, the success rate for target tracking was reduced (Liu and Chen, 2012). Facial attractiveness influences attentionrelated neural activities, such as late positive potential (LPP) which is a late positive component in event-related potential (ERP) waves. One of the potential functions of LPP might be indicating the attentional allocation. From this perspective, researchers found that HA cartoon faces induced a larger LPP than did LA faces (Lu et al., 2014). However, it should be noted that the function of LPP is complex. Whether other functions played important roles in these results should be further investigated.

Why do people pay more attention to HA faces? The perspective of mate selection in evolutionary psychology may provide answers to this question. According to mate selection theory, women may emphasize the social position of potential mates, while men may focus on the attractiveness of potential mates (Dewall and Maner, 2008). In accordance with this theory, studies have shown that the attentional bias to HA faces is strongly affected by biological factors such as the sex of the targets and the hormone level of the observers.
First, the sex of the targets may determine the effect of facial attractiveness on visual attention. Some studies have revealed that men may show a stronger attentional bias toward HA oppositesex faces than women may (Fugita et al., 1977; van Straaten et al., 2010; Valuch et al., 2015; Mitrovic et al., 2018). For example, evidence showed that heterosexual men spent less time fixating on LA faces than on HA opposite-sex faces, while there was no significant difference of fixation time between LA and HA faces among heterosexual women (van Straaten et al., 2010). Neurophysiological evidence also revealed that HA oppositesex faces elicited larger N170 and LPP than LA opposite-sex faces did for heterosexual men (Morgan and Kisley, 2014; Zhang et al., 2018). Further studies have shown that both female and male participants demonstrate attentional bias toward HA female faces but not toward HA male faces (Maner et al., 2003, 2007a). For example, an eye-movement tracking study revealed that participants showed longer total fixation time and first fixation time for HA faces compared with LA faces. Notably, the difference of fixation time between HA and LA faces was larger for female faces than that for male faces (Leder et al., 2010). Consistently, neurophysiological evidence has also suggested that heterosexual women showed a greater difference in LPP response to the HA versus LA same-sex faces than did heterosexual men (Hahn et al., 2016). According to evolutionary theory, women may focus on their potential rivals in mate selection, as these HA rivals are threats for these women in attracting satisfactory mates (Maner et al., 2007b; Maner and Ackerman, 2015). Therefore, women may also show attentional bias to HA female faces. However, HA men may not be valid threats to other men in mate selection. Men may thus not show attentional bias toward HA male faces. Therefore, evolutionary theory predicts that both men and women will show attentional bias toward HA female faces.

Second, the romantic relationship status and hormone level of the observer may also play an important role in determining his/her attentional bias toward HA faces. According to evolutionary theory, it can be hypothesized that compared with non-single individuals, single individuals may allocate more attentional resources to HA individuals. Consistently, studies have revealed less attentional bias toward HA faces among participants who were primed by romantic love or in a committed romantic relationship (Maner et al., 2008; Itier and Batty, 2009). Comparatively, there was stronger correlation between total fixation duration and facial attractiveness in single participants (Leder et al., 2016). Importantly, a longitudinal study found that heterosexual individuals pay less attention to HA faces after building romantic relationships (Koranyi and Rothermund, 2012).

Lastly, evolutionary theory also predicts that women in a period of fertility may pay more attention to HA male faces, as such faces may be considered representative of high genetic quality. Consistently, women near ovulation with high fertility spent more time on looking at HA male faces than women with low fertility did (Anderson et al., 2010). Electrophysiological evidence has also indicated that the visual mismatch negativity (vMMN) induced by HA male faces is greater among heterosexual women in the ovulatory phase than among those in the menstrual phase (Zhang et al., 2018). 
This vMMN is an indicator of automatic attentional processing (Kremláček et al., 2016). Taken together, these findings suggest that biological factors significantly influence attentional bias toward HA faces and support evolutionary theory.

Similar to attentional processing, facial attractiveness may also influence mnemonic processing. However, the effects of facial attractiveness on mnemonic processing are inconsistent. For example, previous studies have found that the memory of HA faces is superior to that of LA faces among heterosexual individuals (Zhang et al., 2016), while another study have found opposite results (Wiese $H$. et al., 2014). Neuroimaging evidence showed that better memory for HA faces reflects stronger functional connectivity between reward-related orbitofrontal cortex (OFC) and memory-related hippocampal regions (Itier and Batty, 2009). Moreover, studies have found that the memory of both HA and LA faces is better than that of moderately attractive faces (Fleishman et al., 1976; Lin et al., 2016). These inconsistent results suggest that the mnemonic processing of facial attractiveness may be affected by complex factors. Nevertheless, evolutionary theory is still the basic theoretical framework for explaining the mnemonic processing of facial attractiveness.

Consistent with evolutionary theory, biological factors, such as the sex of targets and the hormone level of observers, also play important roles in the mnemonic processing of facial attractiveness. First, HA female faces are remembered better than are HA male faces. For example, a study concerning spatial episodic memory revealed that both men and women show better performance in memorizing the locations of HA female faces but not male faces (Becker et al., 2005). Correspondingly, an ERP study revealed higher neural activities in the learning and recognition stages for $\mathrm{HA}$ female faces than for LA female faces among heterosexual individuals (Zhang et al., 2011). All of this evidence suggests that the memory of HA female faces is better than that of LA female faces. Second, the mnemonic processing of facial attractiveness is affected by romantic relationship status. According to evolutionary psychology theory, it can be hypothesized that women who are in love and in stable relationships may have poor memory of HA male faces, which is beneficial for the stability of their romantic relationships. Consistently, women involved in a heterosexual romantic relationship perform worse in memorizing HA male faces than do single women (Karremans et al., 2011). Moreover, heterosexual women in high-quality romantic relationships have more false memories of HA male faces (Watkins et al., 2017).

Unlike attentional processing, the mnemonic processing of facial attractiveness is affected by additional factors. The most important factor is facial characteristics. In addition to facial attractiveness, facial distinctiveness may also affect mnemonic processing (Sarno and Alley, 1997; Wickham and Morris, 2003; Bainbridge et al., 2013). Facial distinctiveness refers to the extent that a face deviates from the average face. Faces with greater distinctiveness have been found to be remembered better (Sarno and Alley, 1997). Although some studies have indicated that attractiveness has a monotonic relationship with distinctiveness, others have suggested that this relationship is U-shaped. For example, some studies have found that facial attractiveness is negatively correlated with facial distinctiveness (Rhodes and Tremewan, 1996; Peskin and Newell, 2004; Rhodes, 2006), indicating lower distinctiveness for HA faces. In contrast, other studies have found that atypical faces (both $\mathrm{HA}$ and LA faces) are more distinctive than are average faces (Perrett et al., 1994; Wickham and Morris, 2003; Mende-Siedlecki et al., 2013). Obviously, a more distinctive face is easier to remember than a less distinctive one. Therefore, mixed results have been found in studies concerning the mnemonic processing of facial attractiveness, without controlling for the factor of distinctiveness.

In summary, existing evidence from behavioral, eyemovement and electrophysiological studies reveal the effect of facial attractiveness in adult faces on the attentional and mnemonic processing. However, only a small number of fMRI studies are designed to investigate the neural mechanisms underlying the attentional and mnemonic processing of facial attractiveness. Usually, a HA face is considered an incentive which may activate the reward circuit involving the nucleus accumbens (NAcc), medial prefrontal, dorsal anterior cingulate and OFC (Senju and Csibra, 2008). An fMRI study found that the activations in OFC were different between HA and LA opposite-sex faces only for men, which was in accordance with the evolutionary theory (Cloutier et al., 2008). Furthermore, researchers investigate how task and attentional state influence the brain activities induced by attractiveness. First, passive viewing HA female faces may activate the NAcc, while subcortical and paralimbic reward regions are activated only when participants were performing an active keypress task (Aharon et al., 2001). Second, brain activities in the ventral striatum were found to increase with facial attractiveness, only when the eye gaze of the face was direct (Kampe et al., 2001). Third, when participants judged facial attractiveness explicitly, neural activities in a widely distributed network involving the ventral occipital, anterior insular, dorsal posterior parietal, inferior dorsolateral, and medial prefrontal cortices were correlated with the level of facial attractiveness, while such associations only remained in ventral occipital region when subjects were not attending explicitly to facial attractiveness (Gredebäck et al., 2010). These fMRI results support the important role of reward circuit in active processing of facial attractiveness. However, it needs further studies to investigate how facial attractiveness biases attention and memory.

\section{COGNITIVE PROCESSING OF FACIAL ATTRACTIVENESS IN INFANT FACES}

The human infant face is a visual stimulus with evolutionary significance. The preference for infant faces is cross-culturally consistent (Esposito et al., 2014). Studies have shown that individuals tend to pay attention to infant faces (Tobias et al., 2007; Lucion et al., 2017). Unlike facial attractiveness in adult faces, the evolutionary value of facial attractiveness in infant faces is not mate selection. The findings concerning infant faces are usually interpreted in evolutionary psychology from the perspective of raising offspring. 
As we previously noted, sex attractiveness and cuteness are two main characteristics of facial attractiveness in adult and infant faces, respectively. Facial cuteness is a facial trait associated with infants and toddlers, such as having large and round eyes, a large head, a round face, and chubby cheeks. These cute facial features construct the infant schema, which could promote the caretaking behavior of observers (Kringelbach et al., 2016). Infant faces with high infant schema traits have been rated as cuter than other infant faces (Borgi et al., 2014). Furthermore, cuter infant faces may elicit stronger positive emotional responses (AlmanzaSepulveda et al., 2018) and stronger caretaking motivation (Glocker et al., 2009a). An electromyography study revealed that unattractive infant faces elicited more corrugator supercilii and levator labii superioris movements which were indicators for negative affect (Bräuer et al., 2005). Therefore, facial attractiveness in infant faces is determined by the facial cuteness.

Facial attractiveness in infant faces can affect the attention and other cognitive processing of such faces. Evidence has revealed that both adults and children may spend more time watching cuter infant faces than less cute infant faces (Hildebrandt and Fitzgerald, 1978; Parsons et al., 2011a; Téglás et al., 2012; Sprengelmeyer et al., 2013; Borgi et al., 2014). Correspondingly, neurophysiological studies have shown that cute infant faces activate the brain regions associated with attention such as the precuneus when participants is asked to assess the cuteness of faces (Glocker et al., 2009b). These results indicate the observer preference for HA infant faces.

According to the perspective in evolutionary psychology of raising offspring, women play more important roles in raising offspring than do men. Therefore, evolutionary theory may predict that women care more about infant faces and are more sensitive to facial attractiveness in infant faces. Studies showed that women who were mothers smiled more frequently to infant faces, and showed larger skin conductance to their own infant (Téglás et al., 2012). Infant faces also elicited stronger and more stable attentional bias for women than for men (Posner and Cohen, 1984). As expected, evidence has further shown that women make greater efforts and spend more time looking at cute infant faces than do men, in a key-press task which allowed them to control the presenting duration of stimuli (Hahn et al., 2013). In addition, women are more sensitive to different levels of cuteness in infant faces and generally have more positive feelings toward infant faces (Glocker et al., 2009a; Sprengelmeyer et al., 2009; Lobmaier et al., 2010; Parsons et al., 2011b; Lehmann et al., 2013). At the neurophysiological level, cute infant faces activate the brain regions of unmarried women, which are related to reward and desire motivation, such as the striatum/NAcc and ventromedial prefrontal cortex (Glocker et al., 2009b; Yin et al., 2017). Taken together, these results indicate that cuter infant face might be a significant reward for women and thus enhance their cognitive processing of these faces.

Consistent with evolutionary theory, other factors related to raising offspring can also affect the cognitive processing of attractiveness in infant faces. First, the hormone level of the observer is an important factor. For example, premenopausal women are more sensitive to facial cuteness than are postmenopausal women and women using oral contraceptives that can artificially raise their hormone levels are more sensitive to facial cuteness (Sprengelmeyer et al., 2009), and women during ovulation are also more sensitive to cuteness differences than are women during the luteal phase (Al-Janabi and Finkbeiner, 2014). In addition, the viewing time of cute infant faces is longer for women whose level of saliva testosterone is high (Hahn et al., 2015a). The second factor is maternal tendencies, which are associated with interest in interacting with babies. Women with stronger maternal tendencies are more likely to press buttons to increase their viewing time of cute infant faces (Hahn et al., 2015b), which is consistent with evolutionary theory. Finally, in addition to the factors of the observers, other factors may influence the willingness to raise offspring and thus the cognitive processing of facial attractiveness in infant faces. For example, infant faces with abnormalities (e.g., cleft lip, strabismus, and hemangioma) are rated less attractive (Lewis et al., 2017; Huffmeijer et al., 2018), and less time is spent by the observers on viewing these faces compared with healthy faces (Parsons et al., 2011b). Another study has shown that mothers of infants with cleft lips allocated significantly less visual attention to their children's faces, especially to the area around the mouth (De Pascalis et al., 2017). Correspondingly, an electrophysiological study revealed lower neural activities (N170 and P2) induced by infant faces with cleft lip compared with healthy infant faces (Huffmeijer et al., 2018).

\section{COMPARISON OF THE COGNITIVE PROCESSING OF FACIAL ATTRACTIVENESS IN ADULT AND INFANT FACES}

According to evolutionary theory, two events are crucial for human reproduction: mate selection and raising offspring. To achieve these two objectives, humans may develop abilities to process stimuli related to these objectives. Visual stimuli, especially faces, provide the main cues for interpersonal communication. Facial attractiveness may be adaptations for mate choice because it signals important aspects of mate quality, such as health, high genetic quality and fertility (Thornhill and Gangestad, 1999; Fink and Penton-Voak, 2002; Rhodes, 2006). It is thus quite natural that humans have cognitive bias toward HA faces. However, the motivations underlying the bias toward HA adult faces and HA infant faces are different. Therefore, there are differences and linkages between the mechanisms underlying the cognitive processing of facial attractiveness in adult and infant faces.

Regarding the common mechanisms, evidence has consistently shown that the cognitive processing of both adult and infant faces can be enhanced with the increase in facial attractiveness (Sui and Liu, 2009; Senese et al., 2013). At the behavioral level, attentional bias toward HA faces has been found in both adult and infant faces, indicated by response time or eye movement (Shimojo et al., 2003; Maner et al., 2007a,b; Dewall and Maner, 2008; Sui and Liu, 2009; Senese et al., 2013; Sprengelmeyer et al., 2013; Borgi et al., 2014; Kou et al., 2016; 
Leder et al., 2016; Mitrovic et al., 2016; Silva et al., 2016). At the neurophysiological level, HA faces may induce higher brain responses than LA faces in brain areas (e.g., striatum/NAcc, precuneus, ventromedial prefrontal cortex) or ERP components (e.g., N170, N2, early posterior negativity (EPN), LPP, vMMN) which may be related to attentional and rewarding processing (Werheid et al., 2007; Glocker et al., 2009b; van Hooff et al., 2011; Chen et al., 2012; Zhang and Deng, 2012; Morgan and Kisley, 2014; Wiese H. et al., 2014; Hahn et al., 2016). These common patterns of cognitive processing are consistent with evolutionary psychology, demonstrating the evolutionary significance of facial attractiveness in both adult and infant faces.

Other than common processing, there are differences between the processing of facial attractiveness in adult faces and that in infant faces. For example, the attentional bias toward HA faces is determined by the sex of the target and of the observer for adult faces and infant faces, respectively. As noted above, these differences may result from the different sources of reproductive motivation. The motivations for adult and infant facial processing derive from mate selection and raising offspring, respectively. As the theoretical frameworks are different, the findings regarding the cognitive processing of facial attractiveness in adult and infant faces should be discussed separately.

Regarding the adult face, evolutionary theory predicts that any faces conducive to choosing the right mate will be considered attractive. Under this theoretical framework, a hypothesis "female beauty captures attention" - is proposed. According to this hypothesis, relative to male faces, HA female faces may be treated with more cognitive bias. There are three reasons that only female faces can capture attention. First, in mate selection, men may want to seek out high-quality potential mates to increase their possibility of producing offspring, and thus, facial attractiveness in female faces is an effective indicator. Second, although women may have the same motivation as men in mate selection, they may place more emphasis on the social resources and social status of the mate (Li et al., 2002; Dewall and Maner, 2008). Relatively, facial attractiveness in male faces is not as important for women as is facial attractiveness in female faces for men. Although women showed attention bias toward HA male faces compared with LA male faces (Silva et al., 2016), the attentional bias toward HA oppositesex faces was significantly stronger for men than that for women (Kleck and Rubenstein, 1975; Fugita et al., 1977; van Straaten et al., 2010; Valuch et al., 2015; Mitrovic et al., 2018). Nevertheless, we should notice that the attractiveness in male faces may be different for women in different situations (e.g., in the ovulatory phase or not). Third, another motivation for women in mate selection is to find potential same-sex rivals to develop appropriate strategies to prevent their current or future partners from being attracted to these HA rivals (Maner et al., 2007b). Given that cognitive resources are limited, compared with allocating cognitive resources to HA male mates, allocating cognitive resources to competitive same-sex rivals makes more sense. Therefore, evolutionary theory predicts that only HA female faces can capture attention from both men and women, and numerous results support this hypothesis (Maner et al., 2003, 2007a; Levy et al., 2008; Lovén et al., 2011).
Although the "female beauty captures attention" hypothesis is supported by a series of studies, other evidence is in favor of another hypothesis - "opposite-sex beauty captures attention" which proposes that both men and women will show selective attention to HA opposite-sex faces (Maner et al., 2003). For example, a study has found that women also show attention bias toward HA male faces compared with LA male faces (Silva et al., 2016). Neurophysiological also revealed that HA opposite-sex faces induced a larger LPP than did LA faces among heterosexual individuals (van Hooff et al., 2011). Nevertheless, although some researchers have found attentional bias toward HA male faces among female observers, they also indicate that such an effect is much weaker than the attentional bias toward HA female faces among male observers (van Straaten et al., 2010; Valuch et al., 2015). The difference between the hypotheses of "female beauty captures attention" and "opposite-sex beauty captures attention" requires further investigation.

Regarding infant faces, evolutionary theory proposes that facial attractiveness or cuteness is a powerful protective mechanism that is beneficial for the survival of infants, through capturing attention, enhancing nurturing intention, eliciting positive affect and protective behaviors, and reducing the possibility of aggression against infants (Borgi et al., 2014). Furthermore, the cuteness of infants can also be an indicator of their health (Golle et al., 2015). According to parentoffspring conflict theory, parents may refrain from investing in newborns until the risk of infant death is reduced to maximize parental investment (Trivers, 1974). Therefore, a cute infant may acquire more nursing and caring compared to a less cute infant. In other words, observers may allocate more cognitive resources to HA infant faces than to LA infant faces. As the motivations are different for the processing of attractiveness in adult and infant faces, the difference in the findings concerning adult and infant faces can be interpreted from different perspectives. First, from the perspective of mate selection, the sex of the face is a determining factor in the processing of facial attractiveness. However, from the perspective of raising offspring, the sex of the face is not important, and instead, the sex of the observer is more crucial in raising offspring. Therefore, there is little evidence showing that the sex of infant faces influences the processing of facial attractiveness. Second, from the perspective of mate selection, men but not women emphasize the physical attractiveness of the mate. Therefore, the effects of facial attractiveness on cognitive processing are greater in male observers than in female observers (Kleck and Rubenstein, 1975; Fugita et al., 1977; van Straaten et al., 2010; Valuch et al., 2015; Mitrovic et al., 2018). However, from the perspective of raising offspring, women play a more important role than do men. Therefore, women are more sensitive to the attractiveness of infant faces than are men (Glocker et al., 2009a; Sprengelmeyer et al., 2009; Lobmaier et al., 2010). Finally, from the perspective of mate selection, romantic relationship status is a key factor in deciding whether to seek a mate or not. Therefore, single individuals show stronger cognitive bias toward HA adult faces compared with non-single individuals (Karremans et al., 2011; Mitrovic et al., 2016). However, from the perspective of raising offspring, the 
hormone levels of women are crucial. Therefore, women with a high hormone level are more sensitive to the attractiveness of infant faces than are women with a low hormone level (Sprengelmeyer et al., 2009).

In summary, in the theoretic framework of evolutionary psychology, a variety of findings concerning facial attractiveness in both adult and infant faces could be explained through reproductive motivations. However, it should be noticed that the evolutionary psychology is not the only theory which could be used to explain experimental findings. Other factors may also influence the cognitive processing of facial attractiveness. For example, the evidence that women showed attentional bias not only to HA male faces but also to HA female faces might potentially be explained from the perspectives that women might be more flexible in sexual orientation or more sensitive in judging the same gender's attractiveness. However, as the current review mainly concerns the evolutionary perspective, these possibilities were not emphasized here and might be discussed in further studies.

\section{SUMMARY AND PROSPECTS}

To summarize, there are common and different mechanisms underlying the cognitive processing of facial attractiveness in adult and infant faces. On the one hand, there are similarities between the processing of facial attractiveness in adult and infant faces. That is, humans generally show cognitive biases to more attractive faces for both adults and infants, which demonstrates the evolutionary significance of facial attractiveness. On the other hand, different factors may determine the cognitive processing of the adult and infant faces. For example, the sex of the face, from the perspective of "female beauty captures attention," is the determinant factor of the processing of adult faces, while the sex of the observer is the determinant factor of the processing of infant faces. The perspectives of mate selection and raising offspring in evolutionary psychology are proposed as potential mechanisms for the processing of facial attractiveness in adult faces and infant faces, respectively. More studies are needed to further elucidate the mechanisms underlying the processing of facial attractiveness.

First, facial cuteness is usually considered the main characteristic of infant facial attractiveness. However, cuteness can also be a characteristic of an adult face. In adult faces, cuteness and attractiveness seem to be two independent characteristics in Western cultures (Geldart, 2010). However, in Eastern cultures such as Japan, a cute female face is considered attractive. Investigations in Japan suggest that cuteness indicates non-sexual attractiveness in female faces for women while it indicates both sexual and non-sexual attractiveness in female faces for men (Cole et al., 2015). Neurophysiological evidence showed that cute adult faces were related to activations in broad regions such as the bilateral insula, bilateral anterior cingulate cortex and right inferior frontal gyrus among Japanese men (Wiese E. et al., 2014). However, whether the mechanisms for the cognitive processing of cuteness are the same as those for the processing of attractiveness in adult faces remains unknown. Therefore, comparisons of these mechanisms between Eastern and Western cultures are an interesting investigation direction.

Second, most findings can be interpreted by evolutionary theory, which emphasizes reproductive motivation in the processing of facial attractiveness. However, under some circumstances, reproduction motivation is relatively weak. Sex orientation is one of the most important factors which may affect the processing of facial attractiveness in adult faces. For example, an eye-movement tracking study revealed that both heterosexual women and homosexual men showed longer total fixation duration and more frequent fixations at HA male faces which were consistent to their sex orientation (Mitrovic et al., 2016). Neurophysiological evidence showed that activities in OFC were higher for HA male faces than HA female faces in heterosexual women and homosexual men, whereas the activities were higher for HA female faces than HA male faces in heterosexual men and homosexual women (Manera et al., 2014). Therefore, other theories should be developed to explain these findings. For example, Sato et al. (2007) proposed a tripartite model of neuroaesthetics which emphasizes cognitive neuroscience framework rather than evolutionary framework. According to this model, aesthetic experiences might emerge from the interaction between emotionvaluation (including reward, emotion and wanting/liking), sensory-motor (involving sensation, perception and motor system), and meaning-knowledge (including expertise, context and culture) neural systems. The stimuli of HA faces may activate these systems in individuals who prefer these stimuli. In addition to the neuroaesthetic model, cognitive behavior model might be another framework to explain the results regarding facial attractiveness. According to the cognitive behavior model of body image disturbance, the cognitive processing for schema-consistent stimuli is facilitated among individuals with maladaptive body selfschemata (Chaminade and Okka, 2013). Accordingly, Kou et al. (2016) found that women with dissatisfaction for their facial attractiveness showed attentional bias toward LA female faces. Other theories besides the evolutionary theory should be further investigated.

Third, in addition to the face, other cues can express one's attractiveness, such as social status, personality, body attractiveness, and voice attractiveness. Some cues are closely related to facial attractiveness and thus may share similar mechanisms. For example, for adults, women's facial attractiveness has been found to be related to body shape (i.e., waist-hip ratio), while men's facial attractiveness has been found to be related to income (Shin et al., 2018). An eye-movement tracking study showed that both men and women spend more time viewing high status male targets than high status female targets (Dewall and Maner, 2008). For infants, it is proposed that positive infant sound and smell may contribute to the cuteness of infants (Kremláček et al., 2016). For example, the syllabic sound produced by 3-month-old boys was rated cuter than that produced by girls, even the boys were labeled with girls' names 
(Nuku and Bekkering, 2008). Further studies are needed to elucidate the mechanisms underlying the processing of these cues and to test evolutionary theory in these studies. In addition, the integration and interaction among different attractiveness cues are also worthy of investigation.

\section{AUTHOR CONTRIBUTIONS}

HK participated in writing the manuscript. QX participated in data collection. TB participated in study design and revising

\section{REFERENCES}

Aharon, I., Etcoff, N., Ariely, D., Chabris, C. F., O'Connor, E., and Breiter, H. C. (2001). Beautiful faces have variable reward value: fMRI and behavioral evidence. Neuron 32, 537-551. doi: 10.1016/s0896-6273(01)00491-3

Al-Janabi, S., and Finkbeiner, M. (2014). Responding to the direction of the eyes: in search of the masked gaze-cueing effect. Attent. Percept. Psychophys. 76, 148-161. doi: 10.3758/s13414-013-0570-6

Almanza-Sepulveda, M. L., Dudin, A., Wonch, K. E., Steiner, M., Feinberg, D. R., Fleming, A. S., et al. (2018). Exploring the morphological and emotional correlates of infant cuteness. Infant Behav. Dev. 53, 90-100. doi: 10.1016/j. infbeh.2018.08.001

Anderson, U. S., Perea, E. F., Becker, D. V., Ackerman, J. M., Shapiro, J. R., Neuberg, S. L., et al. (2010). I only have eyes for you: ovulation redirects attention (but not memory) to attractive men. J. Exp. Soc. Psychol. 46, 804-808. doi: 10.1016/j.jesp.2010.04.015

Bainbridge, W. A., Isola, P., and Oliva, A. (2013). The intrinsic memorability of face photographs. J. Exp. Psychol. Gen. 142, 1323-1334. doi: 10.1037/a0033872

Becker, D. V., Kenrick, D. T., Guerin, S., and Maner, J. K. (2005). Concentrating on beauty: sexual selection and sociospatial memory. Pers. Soc. Psychol. Bull. 31, 1643-1652. doi: 10.1177/0146167205279583

Borgi, M., Cogliati-Dezza, I., Brelsford, V., Meints, K., and Cirulli, F. (2014). Baby schema in human and animal faces induces cuteness perception and gaze allocation in children. Front. Psychol. 5:411. doi: 10.3389/fpsyg.2014.00411

Bräuer, J., Call, J., and Tomasello, M. (2005). All great ape species follow gaze to distant locations and around barriers. J. Comp. Psychol. 119, 145-154. doi: 10.1037/0735-7036.119.2.145

Chaminade, T., and Okka, M. M. (2013). Comparing the effect of humanoid and human face for the spatial orientation of attention. Front. Neurorobot. 7:12. doi: $10.3389 /$ fnbot.2013.00012

Chen, J., Zhong, J., Zhang, Y., Li, P., Zhang, A., Tan, Q., et al. (2012). Electrophysiological correlates of processing facial attractiveness and its influence on cooperative behavior. Neurosci. Lett. 517, 65-70. doi: 10.1016/j. neulet.2012.02.082

Cloutier, J., Heatherton, T. F., Whalen, P. J., and Kelley, W. M. (2008). Are attractive people rewarding? Sex differences in the neural substrates of facial attractiveness. J. Cogn. Neurosci. 20, 941-951. doi: 10.1162/jocn.2008.20062

Cole, G. G., Smith, D. T., and Atkinson, M. A. (2015). Mental state attribution and the gaze cueing effect. Attent. Percept. Psychophys. 77, 1105-1115. doi: 10.3758/s13414-014-0780-6

Collova, J. R., Sutherland, C. A., and Rhodes, G. (2019). Testing the functional basis of first impressions: dimensions for children's faces are not the same as for adults' faces. J. Pers. Soc. Psychol. 117:900. doi: 10.1037/pspa0000167

De Pascalis, L., Kkeli, N., Chakrabarti, B., Dalton, L., Vaillancourt, K., Rayson, H., et al. (2017). Maternal gaze to the infant face: effects of infant age and facial configuration during mother-infant engagement in the first nine weeks. Infant Behav. Dev. 46, 91-99. doi: 10.1016/j.infbeh.2016.12.003

Dewall, C. N., and Maner, J. K. (2008). High status men (but not women) capture the eye of the beholder. Evol. Psychol. 6, 328-341.

Esposito, G., Nakazawa, J., Ogawa, S., Stival, R., Kawashima, A., Putnick, D. L., et al. (2014). Baby, you light-up my face: culture-general physiological responses to infants and culture-specific cognitive judgements of adults. PLoS One 9:e106705. doi: 10.1371/journal.pone.0106705 the manuscript critically. All authors read and approved the final manuscript.

\section{FUNDING}

This research was supported by the New Scientist Training Program of Zunyi Medical University [(2017)5733-014], the Scientific Research Startup Foundation of Zunyi Medical University (F-875 and F-990), and the Research Project of Center for Medical Humanities in Guizhou Universities (2019jd035).

Fink, B., and Penton-Voak, I. (2002). Evolutionary psychology of facial attractiveness. Curr. Dir. Psychol. Sci. 11, 154-158.

Fleishman, J. J., Buckley, M. L., Klosinsky, M. J., Smith, N., and Tuck, B. (1976). Judged attractiveness in recognition memory of women's faces. Percept. Mot. Skills 43, 709-710. doi: 10.2466/pms.1976.43.3.709

Fugita, S. S., Agle, T. A., Newman, I., and Walfish, N. (1977). Attractiveness, selfconcept, and a methodological note about gaze behavior. Pers. Soc. Psychol. Bull. 3, 240-243. doi: 10.1177/014616727700300217

Geldart, S. (2010). That woman looks pretty, but is she attractive? Female perceptions of facial beauty and the impact of cultural labels. Eur. Rev. Appl. Psychol. 60, 79-87. doi: 10.1016/j.erap.2009.12.003

Glocker, M. L., Langleben, D. D., Ruparel, K., Loughead, J. W., Gur, R. C., and Sachser, N. (2009a). Baby schema in infant faces induces cuteness perception and motivation for caretaking in adults. Ethology 115, 257-263. doi: 10.1111/j. 1439-0310.2008.01603.x

Glocker, M. L., Langleben, D. D., Ruparel, K., Loughead, J. W., Valdez, J. N., Griffin, M. D., et al. (2009b). Baby schema modulates the brain reward system in nulliparous women. Proc. Natl. Acad. Sci. U.S.A. 106, 9115-9119. doi: 10.1073/ pnas.0811620106

Golle, J., Probst, F., Mast, F. W., and Lobmaier, J. S. (2015). Preference for cute infants does not depend on their ethnicity or species: evidence from hypothetical adoption and donation paradigms. PLoS One 10:e121554. doi: 10.1371/journal.pone.0121554

Gredebäck, G., Fikke, L., and Melinder, A. (2010). The development of joint visual attention: a longitudinal study of gaze following during interactions with mothers and strangers. Dev. Sci. 13, 839-848. doi: 10.1111/j.1467-7687.2009. 00945.x

Griffey, J. A. F., and Little, A. C. (2014). Infant's visual preferences for facial traits associated with adult attractiveness judgements: data from eye-tracking. Infant Behav. Dev. 37, 268-275. doi: 10.1016/j.infbeh.2014.03.001

Hahn, A. C., Debruine, L. M., Fisher, C. I., and Jones, B. C. (2015a). The reward value of infant facial cuteness tracks within-subject changes in women's salivary testosterone. Horm. Behav. 67, 54-59. doi: 10.1016/j.yhbeh.2014.11.010

Hahn, A. C., Debruine, L. M., and Jones, B. C. (2015b). Reported maternal tendencies predict the reward value of infant facial cuteness, but not cuteness detection. Biology Letters 11, 20140978. doi: 10.1098/rsbl.2014.0978

Hahn, A. C., Symons, L. A., Kredel, T., Hanson, K., Hodgson, L., Schiavone, L., et al. (2016). Early and late event-related potentials are modulated by infant and adult faces of high and low attractiveness. Soc. Neurosci. 11, 207-220. doi: 10.1080/17470919.2015.1059361

Hahn, A. C., Xiao, D., Sprengelmeyer, R., and Perrett, D. I. (2013). Gender differences in the incentive salience of adult and infant faces. Q. J. Exp. Psychol. 66, 200-208. doi: 10.1080/17470218.2012.705860

Hildebrandt, K. A., and Fitzgerald, H. E. (1978). Adults' responses to infants varying in perceived cuteness. Behav. Processes 3, 159-172. doi: 10.1016/03766357(78)90042-6

Huffmeijer, R., Eilander, J., Mileva-Seitz, V. R., and Rippe, R. C. A. (2018). Changes in face-specific neural processing explain reduced cuteness and approachability of infants with cleft lip. Soc. Neurosci. 13, 439-450. doi: 10.1080/17470919.2017. 1340336

Itier, R. J., and Batty, M. (2009). Neural bases of eye and gaze processing: the core of social cognition. Neurosci. Biobehav. Rev. 33, 843-863. doi: 10.1016/j.neubiorev. 2009.02.004 
Kampe, K. K., Frith, C. D., Dolan, R. J., and Frith, U. (2001). Reward value of attractiveness and gaze. Nature 413, 589-589. doi: 10.1038/35098149

Karremans, J. C., Dotsch, R., and Corneille, O. (2011). Romantic relationship status biases memory of faces of attractive opposite-sex others: evidence from a reverse-correlation paradigm. Cognition 121, 422-426. doi: 10.1016/j.cognition. 2011.07.008

Kleck, R. E., and Rubenstein, C. (1975). Physical attractiveness, perceived attitude similarity, and interpersonal attraction in an opposite-sex encounter. J. Pers. Soc. Psychol. 31, 107-114. doi: 10.1037/h0076243

Koranyi, N., and Rothermund, K. (2012). When the grass on the other side of the fence doesn't matter: reciprocal romantic interest neutralizes attentional bias towards attractive alternatives. J. Exp. Soc. Psychol. 48, 186-191. doi: 10.1016/j.jesp.2011.06.012

Kou, H., Su, Y., Bi, T., Gao, X., and Chen, H. (2016). Attentional biases toward facerelated stimuli among face dissatisfied women: orienting and maintenance of attention revealed by eye-movement. Front. Psychol. 7:919. doi: 10.3389/fpsyg. 2016.00919.

Kremláček, J., Kreegipuu, K., Tales, A., Astikainen, P., Põldver, N., Näätänen, R., et al. (2016). Visual mismatch negativity (vMMN): a review and meta-analysis of studies in psychiatric and neurological disorders. Cortex 80, 76-112. doi: 10.1016/j.cortex.2016.03.017

Kringelbach, M. L., Stark, E. A., Alexander, C., Bornstein, M. H., and Stein, A. (2016). On cuteness: unlocking the parental brain and beyond. Trends Cogn. Sci. 20, 545-558. doi: 10.1016/j.tics.2016.05.003

Leder, H., Mitrovic, A., and Goller, J. (2016). How beauty determines gaze! Facial attractiveness and gaze duration in images of real world scenes. i-Perception 7:4.

Leder, H., Tinio, P. P., Fuchs, I. M., and Bohrn, I. (2010). When attractiveness demands longer looks: the effects of situation and gender. Q. J. Exp. Psychol. 63, 1858-1871. doi: 10.1080/17470211003605142

Lehmann, V., Huis In't Veld, E. M., and Vingerhoets, A. J. (2013). The human and animal baby schema effect: correlates of individual differences. Behav. Processes 94, 99-108. doi: 10.1016/j.beproc.2013.01.001

Levy, B., Ariely, D., Mazar, N., Chi, W., Lukas, S., and Elman, I. (2008). Gender differences in the motivational processing of facial beauty. Learn. Motiv. 39, 136-145. doi: 10.1016/j.lmot.2007.09.002

Lewis, J., Roberson, D., and Foulsham, T. (2017). The impact of facial abnormalities and their spatial position on perception of cuteness and attractiveness of infant faces. PLoS One 12:e0180499. doi: 10.1371/journal.pone.018 0499

Li, J., Oksama, L., and Hyönä, J. (2016). How facial attractiveness affects sustained attention. Scand. J. Psychol. 57, 383-392. doi: 10.1111/sjop.12304

Li, N. P., Bailey, J. M., Kenrick, D. T., and Linsenmeier, J. A. (2002). The necessities and luxuries of mate preferences: testing the tradeoffs. J. Pers. Soc. Psychol. 82, 947-955. doi: 10.1037/0022-3514.82.6.947

Lin, T., Lendry, R., and Ebner, N. C. (2016). Face likeability mediates the memoryenhancing effect of face attractiveness in young but not older adults. Memory 24, 1396-1406. doi: 10.1080/09658211.2015.1117109

Lindell, A. K., and Lindell, K. L. (2014). Beauty captures the attention of the beholder. J. Cogn. Psychol. 26, 768-780. doi: 10.1080/20445911.2014.963111

Liu, C. H., and Chen, W. (2012). Beauty is better pursued: effects of attractiveness in multiple-face tracking. Q. J. Exp. Psychol. 65, 553-564. doi: 10.1080/17470218. 2011.624186

Lobmaier, J. S., Sprengelmeyer, R., Wiffen, B., and Perrett, D. I. (2010). Female and male responses to cuteness, age and emotion in infant faces. Evol. Hum. Behav. 31, 16-21. doi: 10.1016/j.evolhumbehav.2009.05.004

Lovén, J., Herlitz, A., and Rehnman, J. (2011). Women's own-gender bias in face recognition memory. Exp. Psychol. 58, 333-340. doi: 10.1027/1618-3169/ a000100

Lu, Y., Wang, J., Wang, L., Wang, J., and Qin, J. (2014). Neural responses to cartoon facial attractiveness: an event-related potential study. Neurosci. Bull. 30, 441-450. doi: 10.1007/s12264-013-1401-4

Lucion, M. K., Oliveira, V., Bizarro, L., Bischoff, A. R., Silveira, P. P., and KauerSant'anna, M. (2017). Attentional bias toward infant faces - Review of the adaptive and clinical relevance. Int. J. Psychophysiol. 114, 1-8. doi: 10.1016/j. ijpsycho.2017.01.008

Maner, J. K., and Ackerman, J. M. (2015). Sexually selective cognition. Curr. Opin. Psychol. 1, 52-56. doi: 10.1016/j.copsyc.2014.11.014
Maner, J. K., Gailliot, M. T., and Dewall, C. N. (2007a). Adaptive attentional attunement: evidence for mating-related perceptual bias. Evol. Hum. Behav. 28, 28-36. doi: 10.1016/j.evolhumbehav.2006.05.006

Maner, J. K., Gailliot, M. T., Rouby, D. A., and Miller, S. L. (2007b). Can't take my eyes off you: attentional adhesion to mates and rivals. J. Pers. Soc. Psychol. 93, 389-401. doi: 10.1037/0022-3514.93.3.389

Maner, J. K., Kenrick, D. T., Becker, D. V., Delton, A. W., Hofer, B., Wilbur, C. J., et al. (2003). Sexually selective cognition: beauty captures the mind of the beholder. J. Pers. Soc. Psychol. 85, 1107-1120. doi: 10.1037/0022-3514.85.6.1107

Maner, J. K., Rouby, D. A., and Gonzaga, G. C. (2008). Automatic inattention to attractive alternatives: the evolved psychology of relationship maintenance. Evol. Hum. Behav. 29, 343-349. doi: 10.3389/fpsyg.2019.01687

Manera, V., Elena, M. R., Bayliss, A. P., and Becchio, C. (2014). When seeing is more than looking: intentional gaze modulates object desirability. Emotion 14, 824-832. doi: $10.1037 / \mathrm{a} 0036258$

Mende-Siedlecki, P., Said, C. P., and Todorov, A. (2013). The social evaluation of faces: a meta-analysis of functional neuroimaging studies. Soc. Cogn. Affect. Neurosci. 8, 285-299. doi: 10.1093/scan/nsr090

Mitrovic, A., Goller, J., Tinio, P. P. L., and Leder, H. (2018). How relationship status and sociosexual orientation influence the link between facial attractiveness and visual attention. PLoS One 13:e0207477. doi: 10.1371/journal.pone.0207477

Mitrovic, A., Tinio, P. P. L., and Leder, H. (2016). Consequences of beauty: effects of rater sex and sexual orientation on the visual exploration and evaluation of attractiveness in real world scenes. Front. Hum. Neurosci. 10:122. doi: 10.3389/ fnhum.2016.00122

Morgan, L. K., and Kisley, M. A. (2014). The effects of facial attractiveness and perceiver's mate value on adaptive allocation of central processing resources. Evol. Hum. Behav. 35, 96-102. doi: 10.1016/j.evolhumbehav.2013.11.002

Nuku, P., and Bekkering, H. (2008). Joint attention: inferring what others perceive (and don't perceive). Conscious. Cogn. 17, 339-349. doi: 10.1016/j.concog.2007. 06.014

Oosterhof, N. N., and Todorov, A. (2008). The functional basis of face evaluation. Proc. Natl. Acad. Sci. U.S.A. 105, 11087-11092. doi: 10.1073/pnas.0805664105

Parsons, C. E., Young, K. S., Kumari, N., Stein, A., and Kringelbach, M. L. (2011a). The motivational salience of infant faces is similar for men and women. Plos One 6, e20632. doi: 10.1371/journal.pone.0020632

Parsons, C. E., Young, K. S., Parsons, E., Dean, A., Murray, L., Goodacre, T., et al. (2011b). The effect of cleft lip on adults' responses to faces: cross-species findings. PLoS One 6:e25897. doi: 10.1371/journal.pone.0025897

Perrett, D. I., May, K. A., and Yoshikawa, S. (1994). Facial shape and judgements of female attractiveness. Nature 368, 239-242. doi: 10.1038/368239a0

Peskin, M., and Newell, F. N. (2004). Familiarity breeds attraction: effects of exposure on the attractiveness of typical and distinctive faces. Perception 33, 147-157. doi: $10.1068 / \mathrm{p} 5028$

Posner, M. I., and Cohen, Y. (1984). Components of visual orienting. Attent. Perform. X 32, 531-556.

Rhodes, G. (2006). The evolutionary psychology of facial beauty. Annu. Rev. Psychol. 57, 199-226. doi: 10.1146/annurev.psych.57.102904.190208

Rhodes, G., and Tremewan, T. (1996). Averageness, exaggeration, and facial attractiveness. Psychol. Sci. 7, 105-110. doi: 10.1111/j.1467-9280.1996. tb00338.x

Sarno, J. A., and Alley, T. R. (1997). Attractiveness and the memorability of faces: Only a matter of distinctiveness? Am. J. Psychol. 110, 81-92.

Sato, W., Okada, T., and Toichi, M. (2007). Attentional shift by gaze is triggered without awareness. Exp. Brain Res. 183, 87-94. doi: 10.1007/s00221-007$1025-\mathrm{x}$

Senese, V. P., De Falco, S., Bornstein, M. H., Caria, A., Buffolino, S., and Venuti, P. (2013). Human infant faces provoke implicit positive affective responses in parents and non-parents alike. PLoS One 8:e80379. doi: 10.1371/journal.pone. 0080379

Senju, A., and Csibra, G. (2008). Gaze following in human infants depends on communicative signals. Curr. Biol. 18, 668-671. doi: 10.1016/j.cub.2008.03.059

Shimojo, S., Simion, C., Shimojo, E., and Scheier, C. (2003). Gaze bias both reflects and influences preference. Nat. Neurosci. 6, 1317-1322. doi: 10.1038/nn1150

Shin, J.-E., Suh, E. M., and Jang, D. (2018). Mate value at a glance: facial attractiveness reveals women's waist-to-hip ratio and men's household income. Pers. Individ. Dif. 135, 128-130. doi: 10.1016/j.paid.2018.07.014 
Silva, A., Macedo, A. F., Albuquerque, P. B., and Arantes, J. (2016). Always on my mind? Recognition of attractive faces may not depend on attention. Front. Psychol. 7:53. doi: 10.3389/fpsyg.2016.00053

Slater, A., Quinn, P. C., Hayes, R., and Brown, E. (2000). The role of facial orientation in newborn infants' preference for attractive faces. Dev. Sci. 3, 181-185. doi: 10.1111/1467-7687.00111

Sprengelmeyer, R., Lewis, J., Hahn, A., and Perrett, D. I. (2013). Aesthetic and incentive salience of cute infant faces: studies of observer sex, oral contraception and menstrual cycle. PLoS One 8:e65844. doi: 10.1371/journal.pone.0065844

Sprengelmeyer, R., Perrett, D. I., Fagan, E. C., Cornwell, R. E., Lobmaier, J. S., Sprengelmeyer, A., et al. (2009). The cutest little baby face:a hormonal link to sensitivity to cuteness in infant faces. Psychol. Sci. 20, 149-154. doi: 10.1111/j. 1467-9280.2009.02272.x

Sui, J., and Liu, C. H. (2009). Can beauty be ignored? effects of facial attractiveness on covert attention. Psychon. Bull. Rev. 16, 276-281. doi: 10.3758/pbr.16.2.276

Sutherland, C. A., Oldmeadow, J. A., Santos, I. M., Towler, J., Burt, D. M., and Young, A. W. (2013). Social inferences from faces: ambient images generate a three-dimensional model. Cognition 127, 105-118. doi: 10.1016/j.cognition. 2012.12.001

Téglás, E., Gergely, A., Kupán, K., Miklósi, Á., and Topál, J. (2012). Dogs’ gaze following is tuned to human communicative signals. Curr. Biol. 22, 209-212. doi: $10.1016 /$ j.cub.2011.12.018

Thornhill, R., and Gangestad, S. W. (1999). Facial attractiveness. Trends Cogn. Sci. 3, 452-460.

Tobias, B., David, S., and Scherer, K. R. (2007). That baby caught my eye... attention capture by infant faces. Emotion 7, 685-689. doi: 10.1037/1528-3542.7.3.685

Trivers, R. L. (1974). Parent-offspring conflict. Curr. Biol. CB 14, 249-264.

Valuch, C., Pflüger, L. S., Wallner, B., Laeng, B., and Ansorge, U. (2015). Using eye tracking to test for individual differences in attention to attractive faces. Front. Psychol. 6:42. doi: 10.3389/fpsyg.2015.00042

Van Duuren, M., Kendell-Scott, L., and Stark, N. (2003). Early aesthetic choices: infant preferences for attractive premature infant faces. Int. J. Behav. Dev. 27, 212-219. doi: 10.1080/01650250244000218

van Hooff, J. C., Crawford, H., and Van Vugt, M. (2011). The wandering mind of men: ERP evidence for gender differences in attention bias towards attractive opposite sex faces. Soc. Cogn. Affect. Neurosci. 6, 477-485. doi: 10.1093/scan/ nsq066

van Straaten, I., Holland, R. W., Finkenauer, C., Hollenstein, T., and Engels, R. C. (2010). Gazing behavior during mixed-sex interactions: sex and attractiveness effects. Arch. Sex. Behav. 39, 1055-1062. doi: 10.1007/s10508-009-9482-x

Watkins, C. D., Nicholls, M. J., Batres, C., Xiao, D., Talamas, S., and Perrett, D. I. (2017). Own attractiveness and perceived relationship quality shape sensitivity in women's memory for other men on the attractiveness dimension. Cognition 163, 146-154. doi: 10.1016/j.cognition.2017.03.007
Werheid, K., Schacht, A., and Sommer, W. (2007). Facial attractiveness modulates early and late event-related brain potentials. Biol. Psychol. 76, 100-108. doi: 10.1016/j.biopsycho.2007.06.008

Wickham, L. H., and Morris, P. E. (2003). Attractiveness, distinctiveness, and recognition of faces: attractive faces can be typical or distinctive but are not better recognized. Am. J. Psychol. 116, 455-468.

Wiese, E., Wykowska, A., and Müller, H. J. (2014). What we observe is biased by what other people tell us: beliefs about the reliability of gaze behavior modulate attentional orienting to gaze cues. PLoS One 9:e94529. doi: 10.1371/journal. pone.0094529

Wiese, H., Altmann, C. S., and Schweinberger, S. R. (2014). Effects of attractiveness on face memory separated from distinctiveness: evidence from event-related brain potentials. Neuropsychologia 56, 26-36. doi: 10.1016/j.neuropsychologia. 2013.12.023

Yin, L., Fan, M., Lin, L., Sun, D., and Wang, Z. (2017). Attractiveness modulates neural processing of infant faces differently in males and females. Front. Hum. Neurosci. 11:551. doi: 10.3389/fnhum.2017.00551

Zhang, S., Wang, H., and Guo, Q. (2018). Sex and physiological cycles affect the automatic perception of attractive opposite-sex faces: a visual mismatch negativity study. Evol. Psychol. 16:1474704918812140. doi: 10.1177/ 1474704918812140

Zhang, Y., Kong, F., Chen, H., Jackson, T., Han, L., Meng, J., et al. (2011). Identifying cognitive preferences for attractive female faces: an event-related potential experiment using a study-test paradigm. J. Neurosci. Res. 89, 18871893. doi: 10.1002/jnr.22724

Zhang, Y., Wei, B., Zhao, P., Zheng, M., and Zhang, L. (2016). Gender differences in memory processing of female facial attractiveness: evidence from eventrelated potentials. Neurocase 22, 317-323. doi: 10.1080/13554794.2016.115 1532

Zhang, Z., and Deng, Z. (2012). Gender, facial attractiveness, and early and late event-related potential components. J. Integr. Neurosci. 11, 477-487. doi: 10. $1142 / \mathrm{S} 0219635212500306$

Conflict of Interest: The authors declare that the research was conducted in the absence of any commercial or financial relationships that could be construed as a potential conflict of interest.

Copyright $(2020 \mathrm{Kou}$, Xie and Bi. This is an open-access article distributed under the terms of the Creative Commons Attribution License (CC BY). The use, distribution or reproduction in other forums is permitted, provided the original author(s) and the copyright owner(s) are credited and that the original publication in this journal is cited, in accordance with accepted academic practice. No use, distribution or reproduction is permitted which does not comply with these terms. 